\title{
THE PATHOLOGY OF ISLETS IN DIABETES
}

\author{
A. K. FOULIS \\ Glasgow
}

Diabetes mellitus is not a disease. It is a syndrome of altered metabolism resulting in, among other things, hyperglycaemia. While there are a number of different diseases which cause this syndrome $99.8 \%$ of diabetic patients in Western countries have either type 1 (insulindependent, juvenile onset) or type 2 (non-insulin-dependent, maturity onset) diabetes. These are quite distinct pathological entities, albeit that many of the complications of the two diseases are common.

\section{NORMAL HUMAN PANCREAS}

Pancreatic islets occupy approximately $2-5 \%$ of the total pancreatic mass in adults. Four distinct endocrine cell types are found within the islets: glucagon-secreting A cells, insulin-secreting B cells, somatostatin-secreting D cells and pancreatic-polypeptide-secreting PP cells. In the body and tail of pancreas approximately $82 \%$ of cells are B cells, $13 \%$ are A cells, $4 \%$ are D cells and $1 \%$ are PP cells. ${ }^{1}$ In the posterior part of the head of pancreas PP cells are much more common (79\%), and this part of the gland, which has a different embryological origin from the rest of the pancreas, is called the PP rich lobe.

\section{THE PANCREAS IN TYPE 1 DIABETES}

In type 1 diabetes there is selective destruction of insulinsecreting $B$ cells within the pancreas. Although patients with type 1 diabetes may present clinically with a relatively acute illness there is evidence that the destruction of $B$ cells proceeds very slowly and may well have been present for years before diagnosis. ${ }^{2}$ Only when approximately $80 \%$ of the B cells have been destroyed will the patient decompensate metabolically.

If the pancreas of a patient who has had the disease for many years is examined, virtually no B cells will be found and the islets will consist only of A, D and PP cells. By contrast, at clinical presentation of the disease, there are essentially three morphological types of islet. Firstly, approximately $70 \%$ of the islets are devoid of B cells ('insulin deficient islets') (Fig. 1). Secondly, there are

Correspondence to: A. K. Foulis, Department of Pathology, Royal Infirmary, Glasgow G4 OSF, UK. islets which are histologically normal and which have a normal complement of B cells. Thirdly, there are islets which show signs of chronic inflammation and which exhibit a variable reduction in the number of $B$ cells (Fig. 2). ${ }^{3}$ This inflammatory process, associated with the selective destruction of B cells, is termed insulitis. ${ }^{4}$

In a study of a single case, in which frozen material was available, the majority of lymphocytes in the insulitis infiltrate were $\mathrm{T}$ cells, with a predominance of those

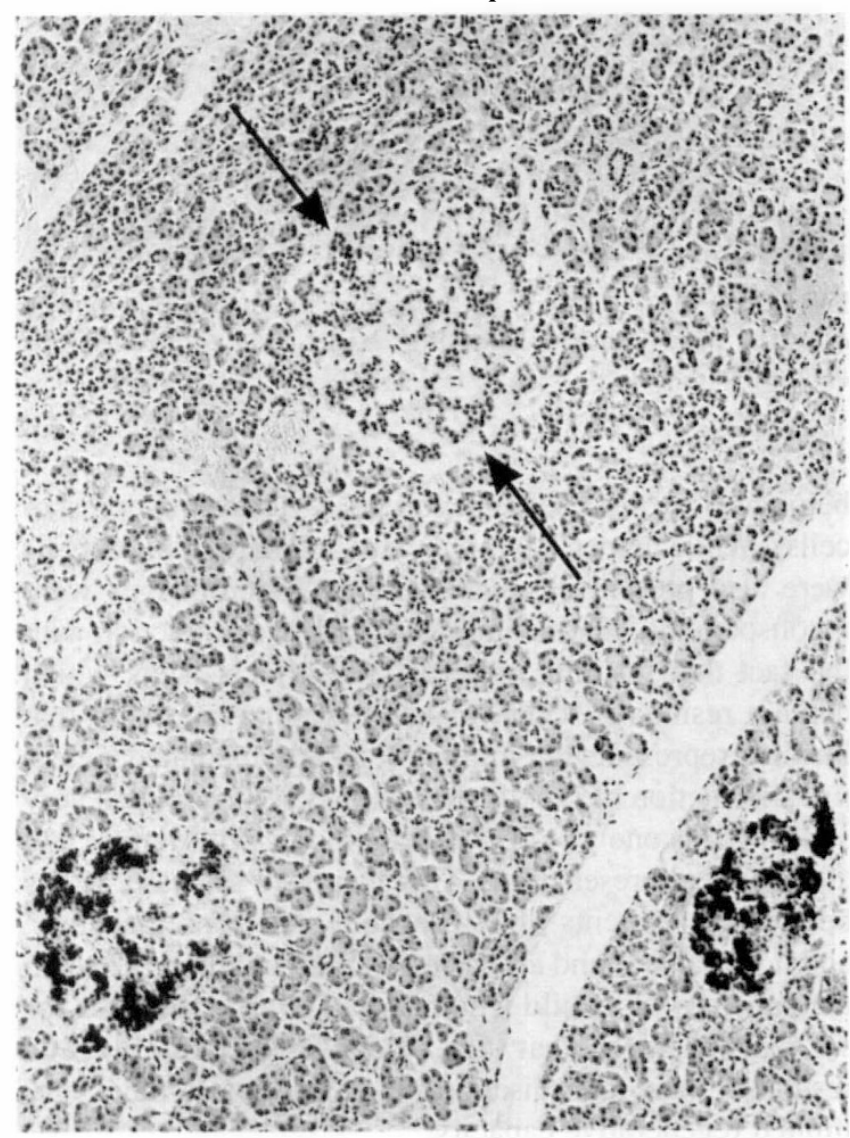

Fig. 1. Pancreas from a patient with newly diagnosed type 1 diabetes. This has been immunostained for insulin. The two islets at the bottom of the photograph contain B cells, but the islet at the top (arrov'ed) does not. Islets in long-standing type 1 diabetes are virtually all insulin deficient. $(\times 50)$ 


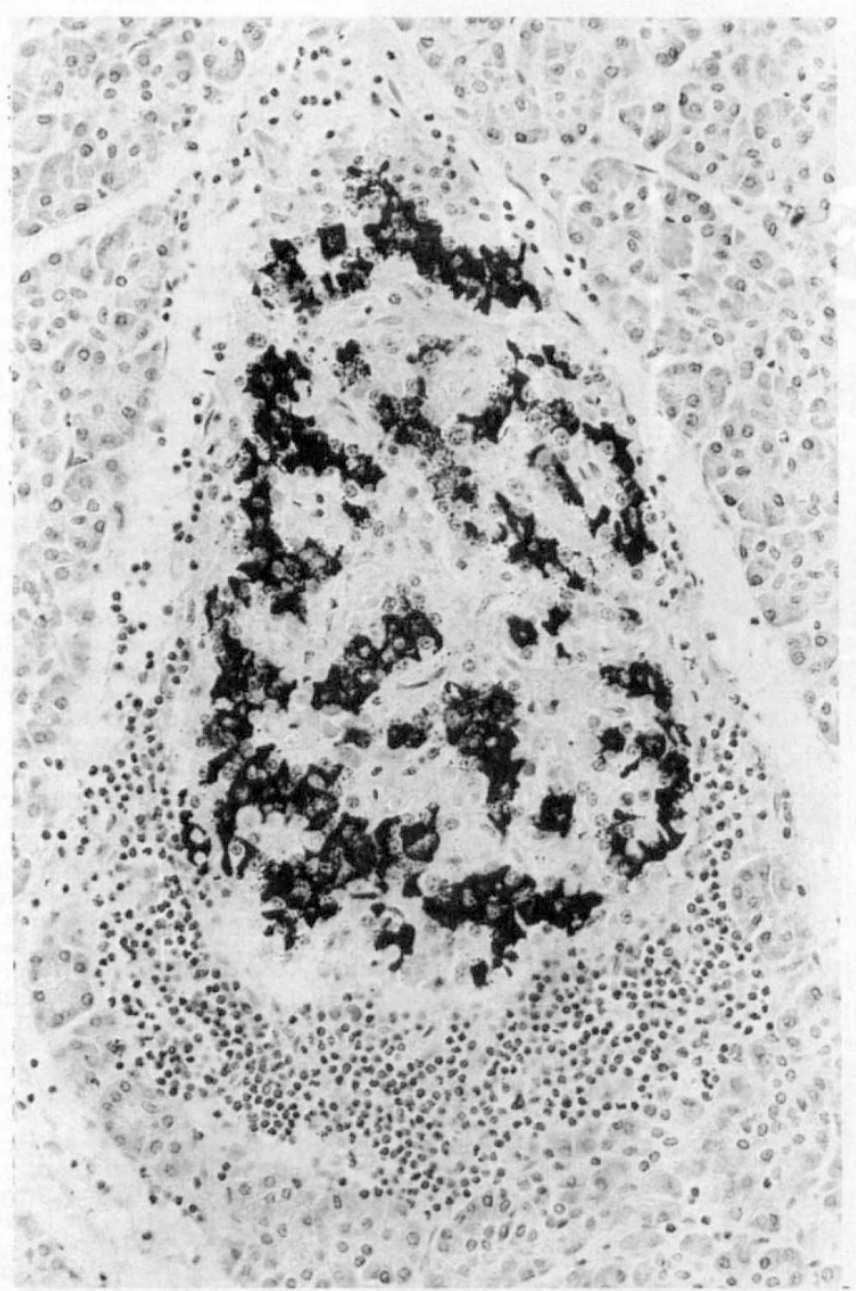

Fig. 2. Insulitis. There is a heavy lymphocytic infiltrate affecting this insulin-containing islet. (Immunostained for insulin, $\times 222$ )

belonging to the CD8 phenotype (cytotoxic/suppressor cells). While helper T lymphocytes and natural killer cells were also present in small numbers macrophages were inconspicuous. ${ }^{5}$ These findings, taken in conjunction with the fact that insulitis preferentially affects islets which contain residual B cells, makes it seem very likely that insulitis represents the immunologically mediated, selective destruction of B cells. If loss of B cells is truly a very slow process one would expect to find insulitis before and after clinical presentation. This is indeed the case. It has been seen in patients who were thought to be prediabetic ${ }^{6.7}$ and it was also found affecting insulin-containing islets in the pancreas of a child who had had clinical diabetes for six years. ${ }^{3}$ The fact that such a slow destruction of B cells results in their total disappearance testifies to their very limited regenerative capacity.

There is now good evidence that type 1 diabetes represents an autoimmune disease in which the B cell is targeted for destruction. ${ }^{8}$ Several immunopathological abnormalities in the islets have been described which may help to point to the pathological process leading to autoimmunity.

\section{Aberrant Expression of Class II MHC by B Cells}

There is a marked genetic component to the development of diabetes which is linked to the class II major histocompatibility complex (MHC) genes (HLA-DR, -DP and -DQ in man). ${ }^{9}$ The proteins coded by these genes are expressed on the surface of certain cells and are intimately involved in antigen presentation. Normally $\mathrm{T}$ helper lymphocytes, the cells which initiate the immune response, only recognise the antigen to which they are directed if the antigen is presented to them by cells which express class II MHC molecules. Many cells in the body, including pancreatic B cells, do not normally express class II MHC molecules. Thus, even if there were potentially autoreactive $\mathrm{T}$ lymphocytes present which could recognise antigens on these cells, they would not react because the antigens would not be 'presented' to them in the context of class II MHC. This may be one mechanism of tolerance. Bottazzo et al. ${ }^{10}$ proposed that one event which may provoke organ-specific autoimmunity would be aberrant expression of class II MHC by target cells, such as pancreatic B cells. This could theoretically convert them into antigen presenting cells, capable of presenting their own surface antigens to potentially autoreactive T lymphocytes. Several authors have now confirmed that aberrant expression of class II MHC by $\mathrm{B}$ cells (but not A, D or PP cells) is an abnormality unique to type 1 diabetes, being absent in all other pancreatic diseases thus far examined (Fig. 3). ${ }^{5,11-13}$

\section{Hyperexpression of Class I MHC}

Whilst helper $\mathrm{T}$ lymphocytes can only recognise the antigen to which they are directed in the context of class II MHC molecules, cytotoxic T lymphocytes, which are the cells most likely to be responsible for destroying islet $\mathrm{B}$ cells, recognise antigen only in the presence of class I MHC (HLA-A, -B and -C in man). Islet B cells, in common with most nucleated cells in the body, normally express these molecules. However, hyperexpression of class I MHC molecules may make them more susceptible to the action of cytotoxic T lymphocytes.

In pancreases from patients with recent onset type 1 diabetes the great majority of islets containing residual $\mathrm{B}$ cells hyperexpressed class $1 \mathrm{MHC}$ whilst islets which were deficient in $B$ cells were not thus affected. Within affected islets A, D and PP cells, as well as B cells, hyperexpressed class I MHC. As with aberrant expression of class II MHC by B cells this abnormality appeared unique to type 1 diabetes. ${ }^{12}$

\section{Interferon-alpha in B Cells}

As has been described above, A and D cells hyperexpressed class I MHC when they were adjacent to B cells in insulin-containing islets. However, when they were physically divorced from B cells, in insulin-deficient islets, they ceased to hyperexpress this product. This, in conjunction with the fact that many islets which hyperexpressed class.I MHC did not appear to be inflamed, suggested that the B cells may be secreting some factor which could cause adjacent endocrine cells to hyperexpress class 


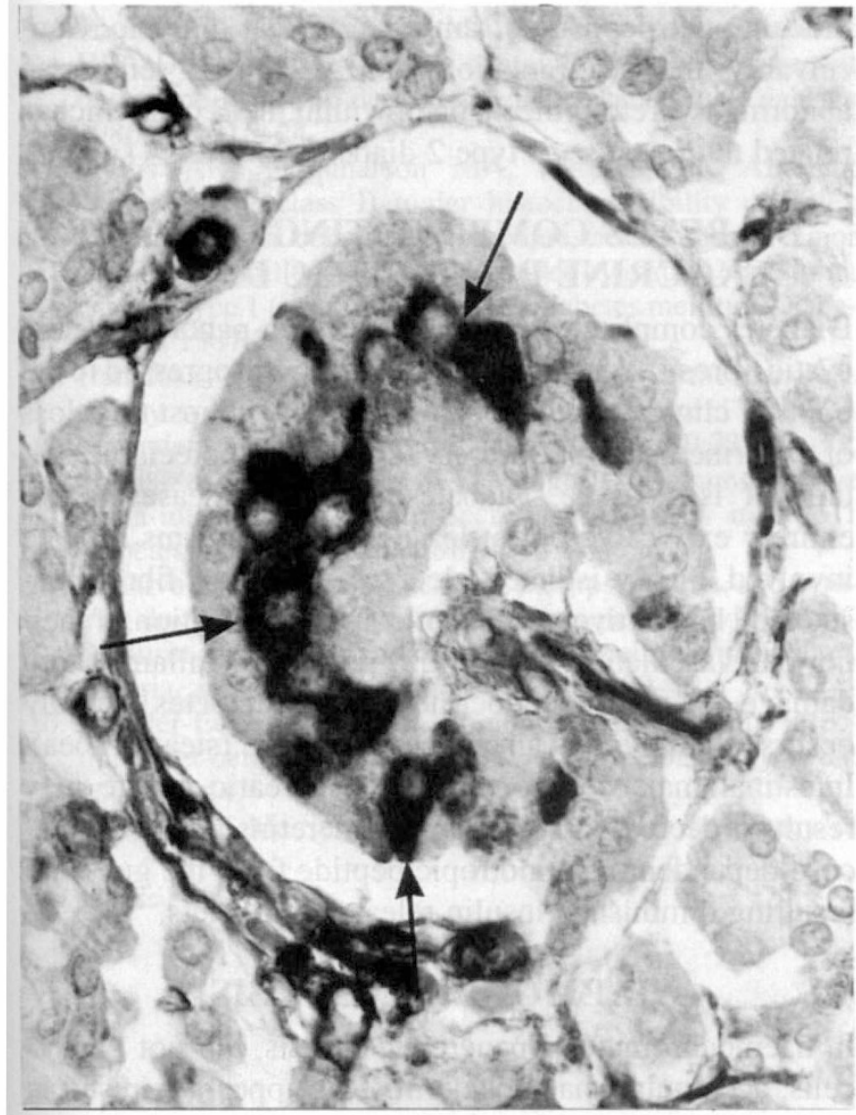

Fig. 3. New'ly diagnosed type 1 diabetes. This islet has been immunostained for class II MHC. Several endocrine cells express this product (arrowed). Double stains showed that B cells but not $A$ or D cells were affected. $(\times 780)$

I MHC. One substance capable of doing this in vitro is interferon-alpha. In an immunohistochemical study of 37 diabetic pancreases immunoreactive interferon-alpha, confined to B cells, was found in $93 \%$ of islets which hyperexpressed class I MHC but only in $0.4 \%$ of those showing no hyperexpression (Fig. 4). Among 80 pancreases from non-diabetic patients or patients with type 2 diabetes significant numbers of $\mathrm{B}$ cells containing interferon-alpha were present only in 4 cases of acute infantile Coxsackie viral pancreatitis. ${ }^{14}$ This raised the possibility that $\mathrm{B}$ cells in diabetes could be infected by a virus. Serological studies of newly diagnosed patients with type 1 diabetes have also suggested that viral infection could be implicated in the pathogenesis of the disease. ${ }^{15-17}$

\section{Insights into the Possible Pathogenesis of Type 1 Diabetes}

The concordance rate in monozygotic twins for autoimmune diabetes is no more than $50 \%$, indicating that environmental factors as well as genetic susceptibility play a role in the pathogenesis of the disease. Chief among the environmental suspects are viruses, particularly Coxsackie, mumps and rubella viruses. ${ }^{15-17}$ The finding that $\mathrm{B}$ cells in both diabetes and Coxsackie viral pancreatitis express interferon-alpha raises the possibility that $\mathrm{B}$ cells are chronically infected by a virus in diabetes. This infection is not likely to be cytopathic, given the long time required for destruction of $\mathrm{B}$ cells in the disease. However, if a 'slow' viral infection provoked the B cells to secrete interferon-alpha this might cause hyperexpression of class I MHC by islet endocrine cells. In the absence of a genetic predisposition perhaps nothing further would happen. However, it is conceivable that either the virus alone, or the virus plus cytokines, such as interferon-gamma and tumour necrosis factor, released in an inflammatory infiltrate, may cause B cells to express class II MHC in per-
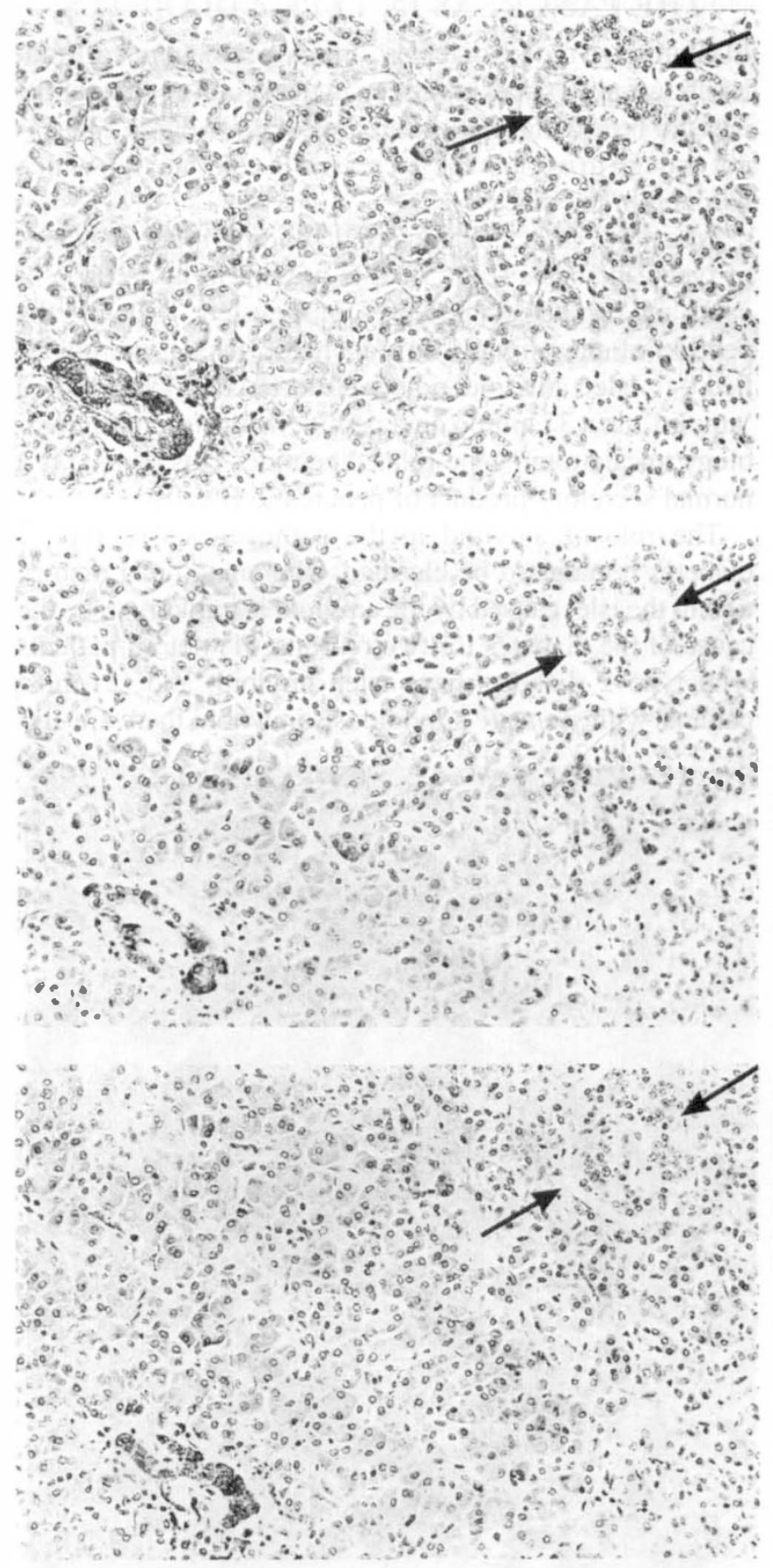

Fig. 4. Nen'ly diagnosed type 1 diabetes. The islet in the bottom left of the photograph still has residual B cells which contain insulin (middle) and express interferon-alpha (bottom). This islet hyperexpresses class I MHC (top). This contrasts with the insulin-deficient islet in the top right of the photograph (arrowed) which neither expresses interferon-alpha nor hyperexpresses class I MHC. (×160) 
sons carrying the genetic susceptibility for diabetes. It is proposed that aberrant expression of class II MHC by B cells is a crucial pathogenic event in diabetes, causing induction of autoimmunity and eventually destruction of the insulin-secreting cells in a cell-mediated fashion. This hypothesis suggests that the development of diabetes is a 'multistep' process involving both environmental and genetic components. ${ }^{18}$

\section{THE PANCREAS IN TYPE 2 DIABETES}

In contrast to type 1 diabetes, immunocytochemical studies show only a small reduction in the B-cell content of the islets of type 2 diabetic pancreases of up to $30 \% .{ }^{19}$ This is not enough to explain the decreased B-cell function which is found in the disease.

Islet amyloid is a characteristic finding in the pancreas of type 2 diabetes (Fig. 5). It is present in up to $90 \%$ of cases and can affect up to $80 \%$ of the islets. Recently the peptide of the amyloid in pancreatic islets was identified. ${ }^{20.21}$ Islet amyloid polypeptide (IAPP) (also called 'amylin') is a 37 amino acid peptide with structural homology to calcitonin gene-related peptide. This peptide is a normal secretory product of pancreatic B cells.

The role of amyloid in the pathogenesis of type 2 diabetes remains to be clarified. The anatomical disruption to the islet caused by the amyloid is unlikely to be the cause of the diabetes for two reasons: (1) not all patients with type 2 diabetes have islet amyloid, and (2) most patients with systemic amyloidosis in whom there are sig-

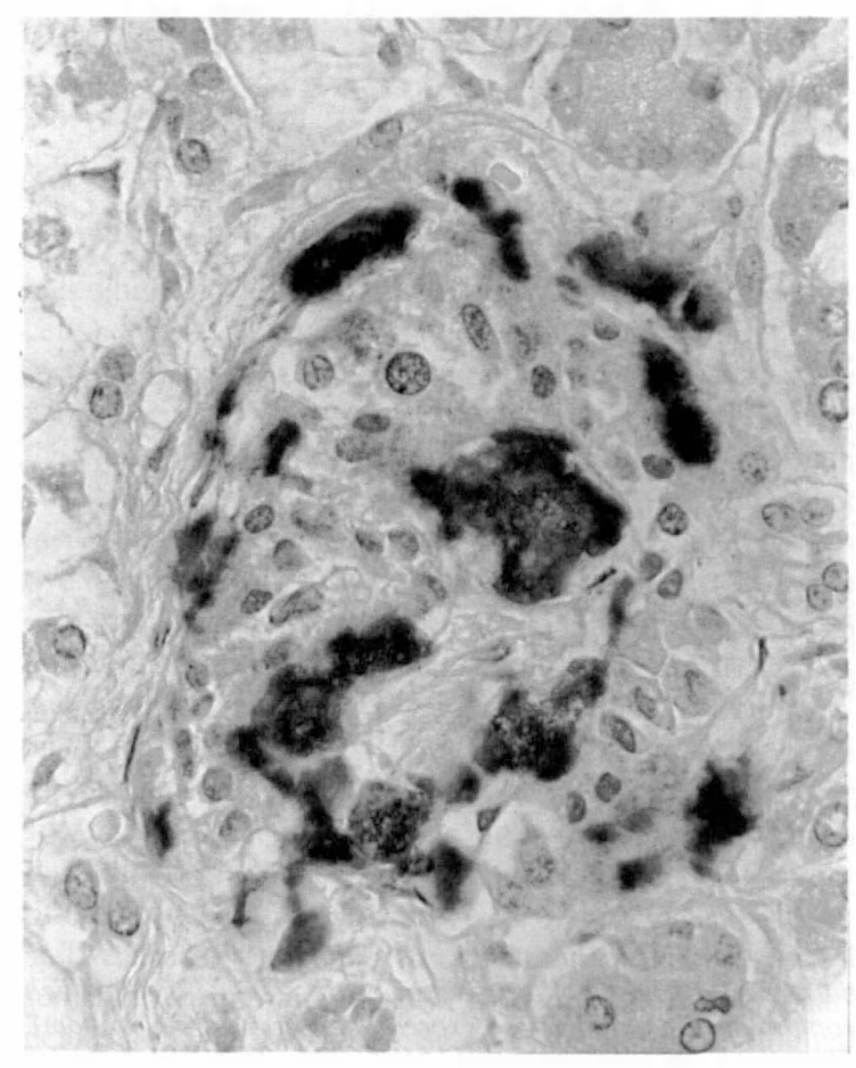

Fig. 5. Amyloid in an islet from a patient with type 2 diabetes. The islet amyloid polypeptide (amylin) is immunostained black. ( $\times 500$; in conjunction with Dr. Anne Clark). nificant islet deposits of amyloid are not diabetic. Conceivably the accumulation of IAPP may reflect an abnormal secretory mechanism within the B cell which is related to the cause of type 2 diabetes.

\section{DIABETES COMPLICATING PRIMARY EXOCRINE PANCREATIC DISEASE}

Diabetes complicates chronic calcifying pancreatitis and cystic fibrosis. When these diseases have progressed to the point of clinical diabetes there is usually almost total loss of exocrine tissue but preservation of an appreciable volume of islet tissue. Diabetes in these diseases is not entirely explicable but at least two mechanisms may be involved. Firstly, isolated islets surrounded by fibrous tissue may be relatively ischaemic due to disruption of their normal vasculature during the preceding inflammatory damage. Secondly, endocrine failure (diabetes) usually occurs when there is also exocrine failure (steatorrhoea). Intestinal malabsorption due to pancreatic failure may result in decreased secretion of 'incretins', such as glucose-dependent insulinotropic peptide from the gut, with resulting diminished insulin release from islets. ${ }^{22}$

\section{HAEMOCHROMATOSIS}

In haemochromatosis pancreatic B cells, but not $\mathrm{A}$ or $\mathrm{D}$ cells, accumulate haemosiderin. This appears to result in toxic damage, impairing the B cells' ability to synthesise and secrete insulin. ${ }^{23}$

Mrs. J Cramb kindly typed the manuscript.

Key words: Diabetes, Pathology.

\section{REFERENCES}

1. Stefan Y et al. Quantitation of endocrine cell content in the pancreas of nondiabetic and diabetic humans. Diabetes 1982;31:694-700.

2. Tarn AC, et al. Type 1 (insulin-dependent) diabetes: a disease of slow clinical onset? BMJ 1987;294:342-5.

3. Foulis AK, et al. The histopathology of the pancreas in type 1 (insulin-dependent) diabetes mellitus: a 25-year review of deaths in patients under 20 years of age in the United Kingdom. Diabetologia 1986;29:267-74.

4. Gepts W. Pathologic anatomy of the pancreas in juvenile diabetes mellitus. Diabetes 1965;14:619-33.

5. Bottazzo GF, et al. In situ characterization of autoimmune phenomena and expression of HLA molecules in the pancreas in diabetic insulitis. N Engl J Med 1985;313:353-60.

6. Foulis AK, Jackson R, Farquharson MA. The pancreas in idiopathic Addison's disease: a search for a prediabetic pancreas. Histopathology 1988;12:481-90.

7. Foulis AK, McGill M, Farquharson MA. Insulitis in type 1 (insulin-dependent) diabetes mellitus in man: macrophages, lymphocytes and interferon-gamma containing cells. J Pathol 1991;165:97-103.

8. Bottazzo GF. Beta-cell damage in diabetic insulitis: are we approaching a solution? Diabetologia 1984;26:241-9.

9. Wolf E, Spencer KM, Cudworth AG. The genetic susceptibility to type 1 (insulin-dependent) diabetes: analysis of the HLA-DR association. Diabetologia 1983;24:224-30.

10. Bottazzo GF, et al. Role of aberrant HLA-DR expression and antigen presentation in induction of endocrine autoimmunity. Lancet 1983;2:1115-8. 
11. Foulis AK, Farquharson MA. Aberrant expression of HLA-DR antigens by insulin containing beta cells in recent onset type 1 (insulin-dependent) diabetes mellitus. Diabetes 1986;35:1215-24.

12. Foulis AK, Farquharson MA, Hardman R. Aberrant expression of class II major histocompatibility complex molecules by B cells and hyperexpression of class I major histocompatibility complex molecules by insulin containing islets in type 1 (insulin-dependent) diabetes mellitus. Diabetologia 1987;30:333-43.

13. Hanafusa $\mathrm{T}$, et al. Examination of islets in the pancreas biopsy specimens from newly diagnosed type 1 (insulindependent) diabetic patients. Diabetologia 1990;33:105-11.

14. Foulis AK, Farquharson MA, Meager A. Immunoreactive alpha-interferon in insulin-secreting beta cells in type 1 diabetes mellitus. Lancet 1987;2:1423-7.

15. Banatvala JE, et al. Coxsackie B, mumps, rubella, and cytomegalovirus specific IgM responses in patients with juvenile-onset insulin-dependent diabetes mellitus in Britain, Austria, and Australia. Lancet 1985;1:1409-12.

16. Mueller-Eckhardt G, et al. Complications of mumps infection, islet-cell antibodies, and HLA. Immunobiology 1984; 167:338-44.

17. Forrest JM, Menser MA, Burgess JA. High frequency of diabetes mellitus in young adults with congenital rubella. Lancet 1971;2:332-4.

18. Foulis AK. Lawrence Lecture: In type 1 diabetes, does a non-cytopathic viral infection of insulin-secreting B-cells initiate the disease process leading to their autoimmune destruction? Diabetic Med 1989;6:666-74.

19. Clark A, et al. Islet amyloid, increased A-cells, reduced B-cells and exocrine fibrosis: quantitative changes in the pancreas in type 2 diabetes. Diabetes Res 1988;9:151-9.

20. Westermark P, et al. Amyloid fibrils in human insulinoma and islets of Langerhans in the diabetic cat are derived from a neuropeptide-like protein also present in normal islet cells. Proc Natl Acad Sci USA 1987;84:3881-5.

21. Clark A, et al. Islet amyloid formed from diabetes-associated peptide may be pathogenic in type 2 diabetes. Lancet 1987;1:231-4.

22. Ebert R, Creutzfeldt W. Reversal of impaired GIP and insulin secretion in patients with pancreatogenic steatorrhoea following enzyme substitution. Diabetologia 1980; 19:198-204.

23. Rahier J, et al The haemochromatotic human pancreas: a quantitative immunohistochemical and ultrastructural study. Diabetologia 1987;30:5-12. 